\title{
Highly Hybridizable Spherical Nucleic Acids by Tandem Glutathione Treatment and Polythymine Spacing
}

Jing Sun, ${ }^{\dagger}$ Dennis Curry, ${ }^{\star}$ Qipeng Yuan,${ }^{\dagger}$ Xu Zhang, ${ }^{*}{ }^{\star \dagger}$ Hao Liang, ${ }^{* \dagger}$

†State Key laboratory of Chemical Resource Engineering, Beijing University of Chemical Technology, Beijing, P. R. China.

*Verschuren Centre of for Sustainability in Energy and the Environment, Cape Breton University, 1250 Grand Lake Road, Sydney, Nova Scotia, Canada.

E-mail address: Xu_Zhang@,cbu.ca. lianghao@mail.buct.edu.cn. 


\section{Supporting data:}

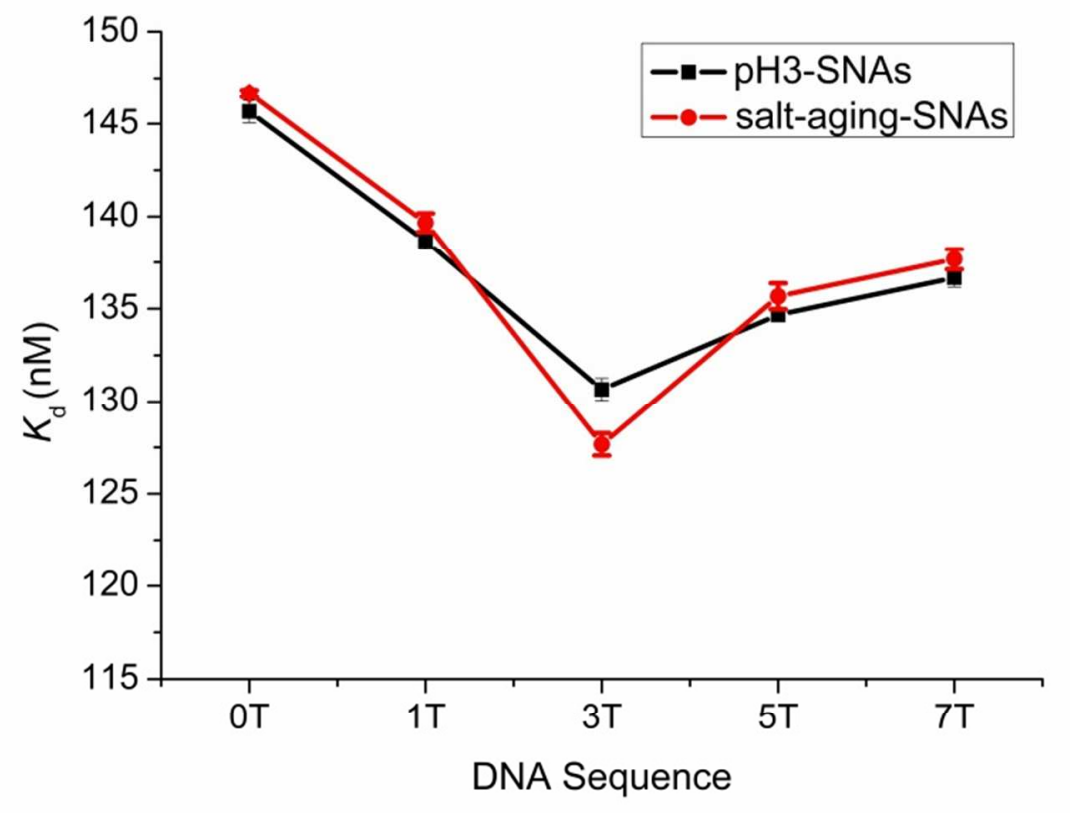

Figure S1. Comparison of the binding constants of complementary DNA sequences (DNA 16) towards SNAs prepared with $\mathrm{pH} 3$ method (pH3-SNAs) and salt-aging procedure (salt-agingSNAs).
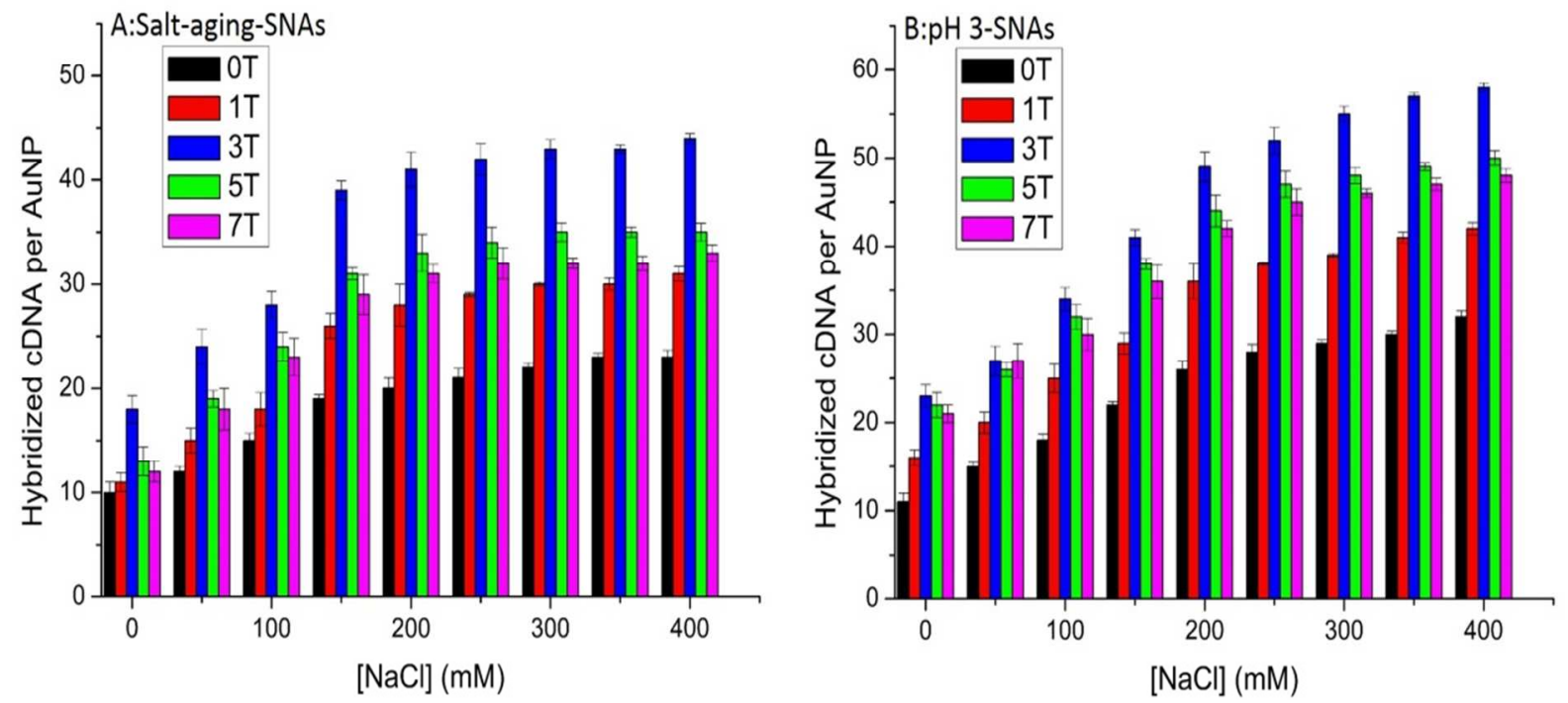

Figure S2. The number of complementary DNA strands that were hybridized on each SNA as a function of salt concentration. 


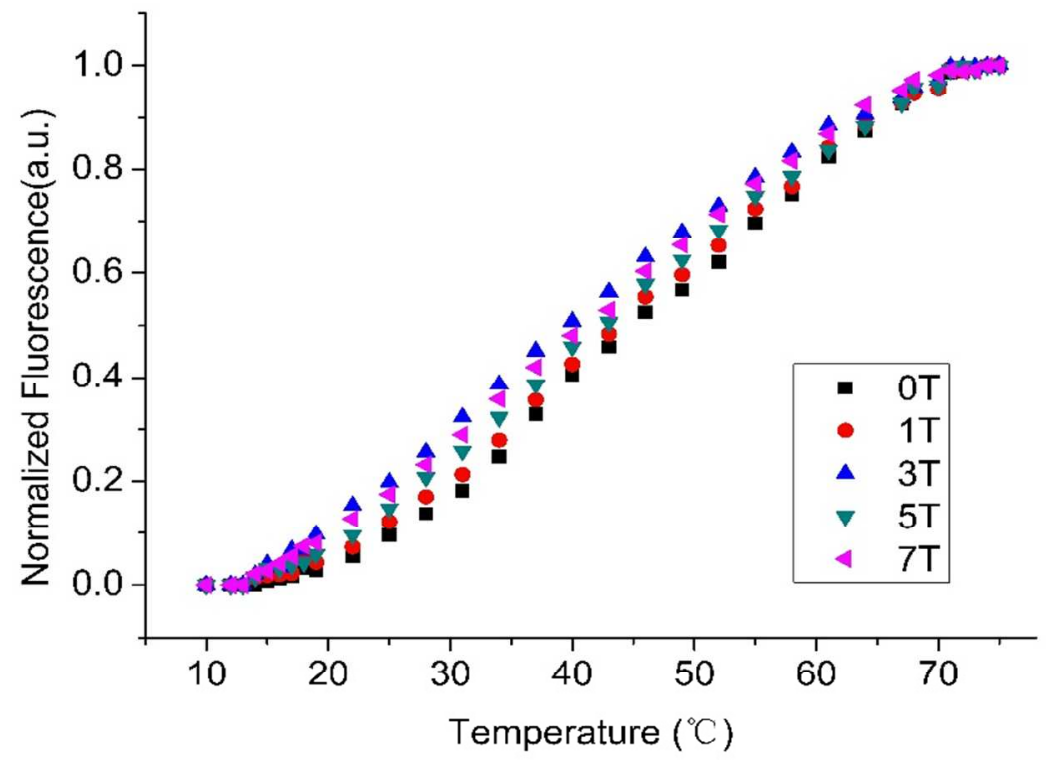

Figure S3. The normalized dehybridization melting curves for SNAs synthesized with thiolated DNA having different polyT-spacer lengths (DNA 1-5 in Table 1).

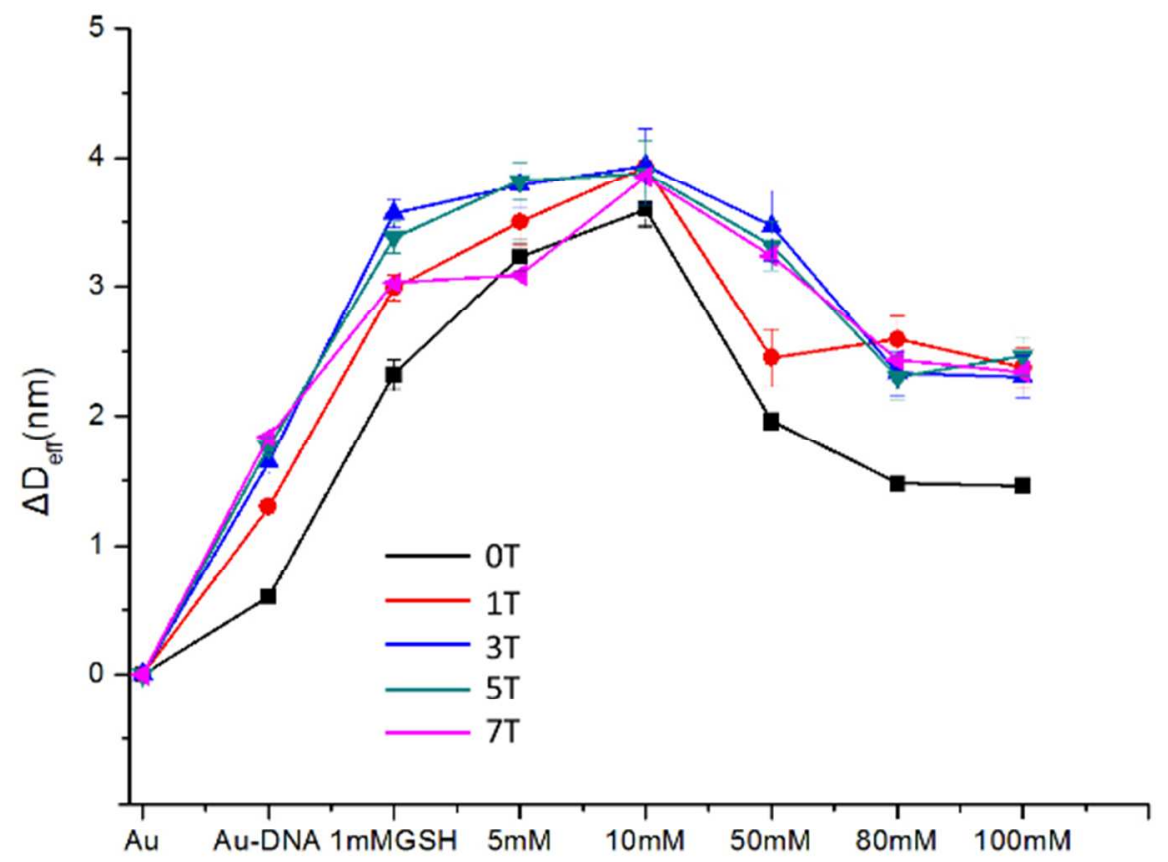

Figure S4. $\Delta \mathrm{D}_{\text {eff, }}$ the hydrodynamic length increase of the adsorbed thiolated DNAs (with different polyT spacers) relative to their theoretical length in free form, as a function of the GSH concentration (1mM-100mM) for 10-min treatment. 

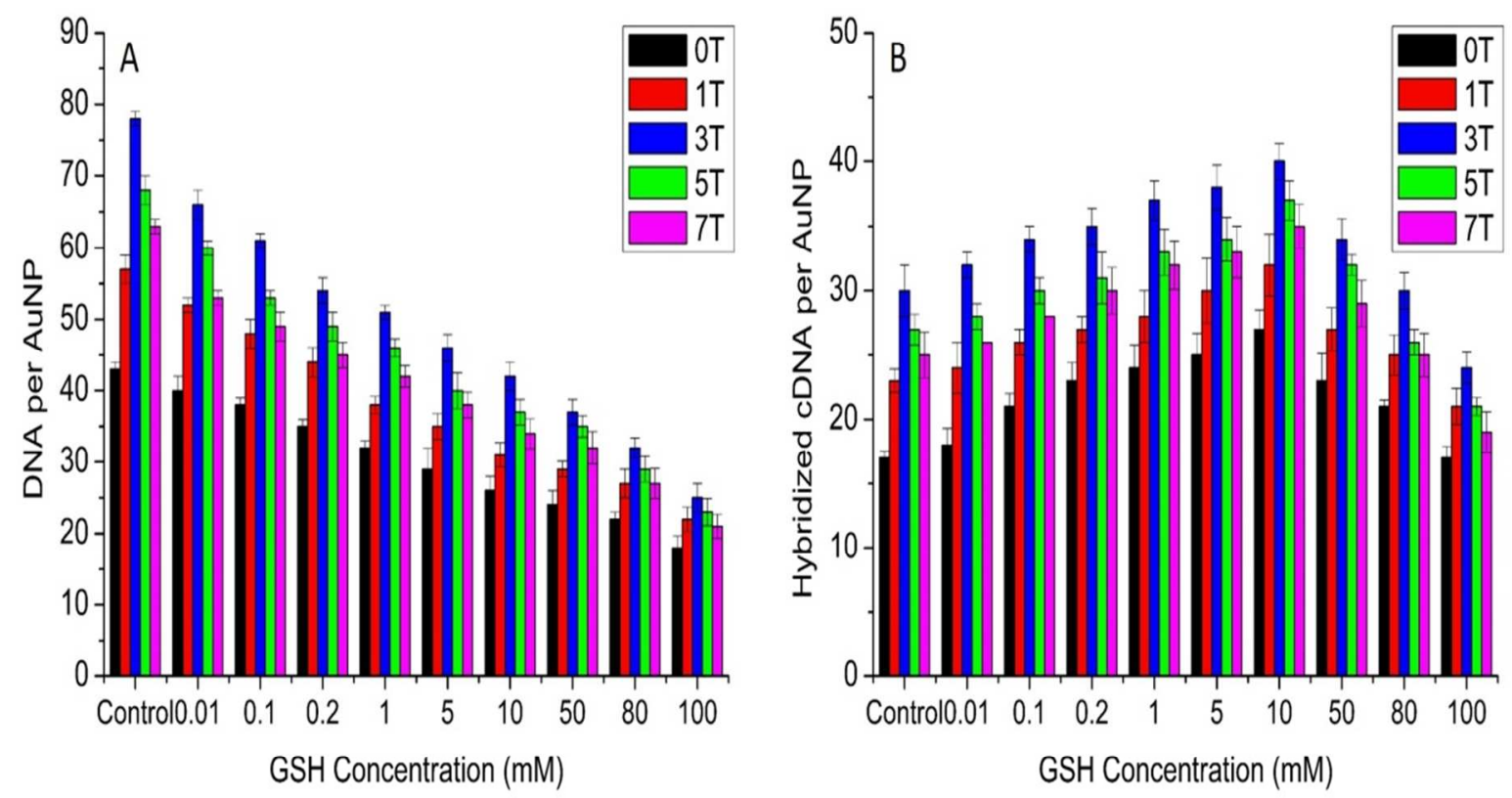

Figure S5. The effect of GSH concentrations (for a 10 min treatment) on the number of adsorbed SH-DNA (A) and hybridized cDNA (B) of each pH3-SNA.
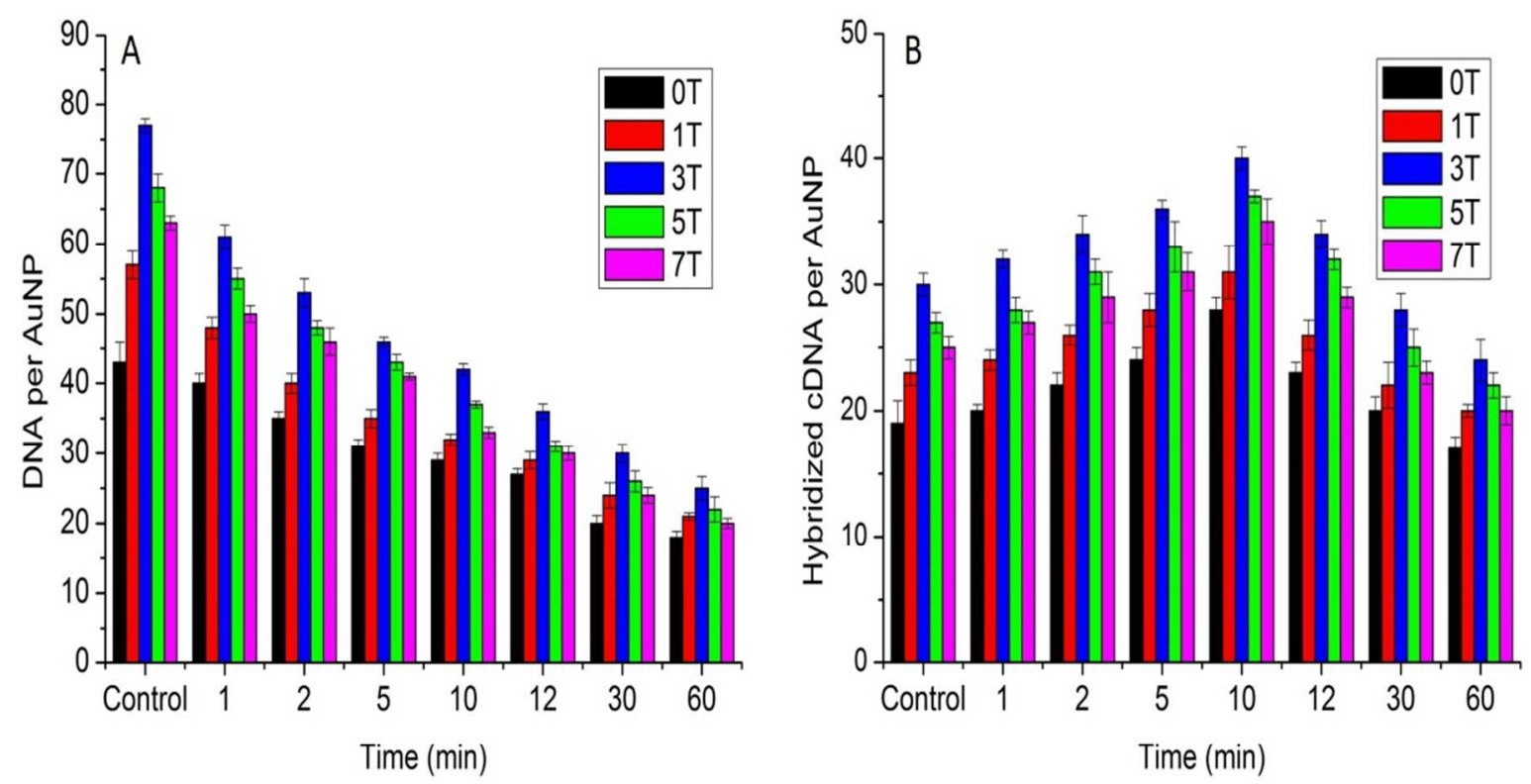

Figure S6. The number of adsorbed thiolated DNAs (A) and hybridized cDNAs (B) on each $\mathrm{pH} 3-\mathrm{SNA}$ as the function of GSH treatment time. 


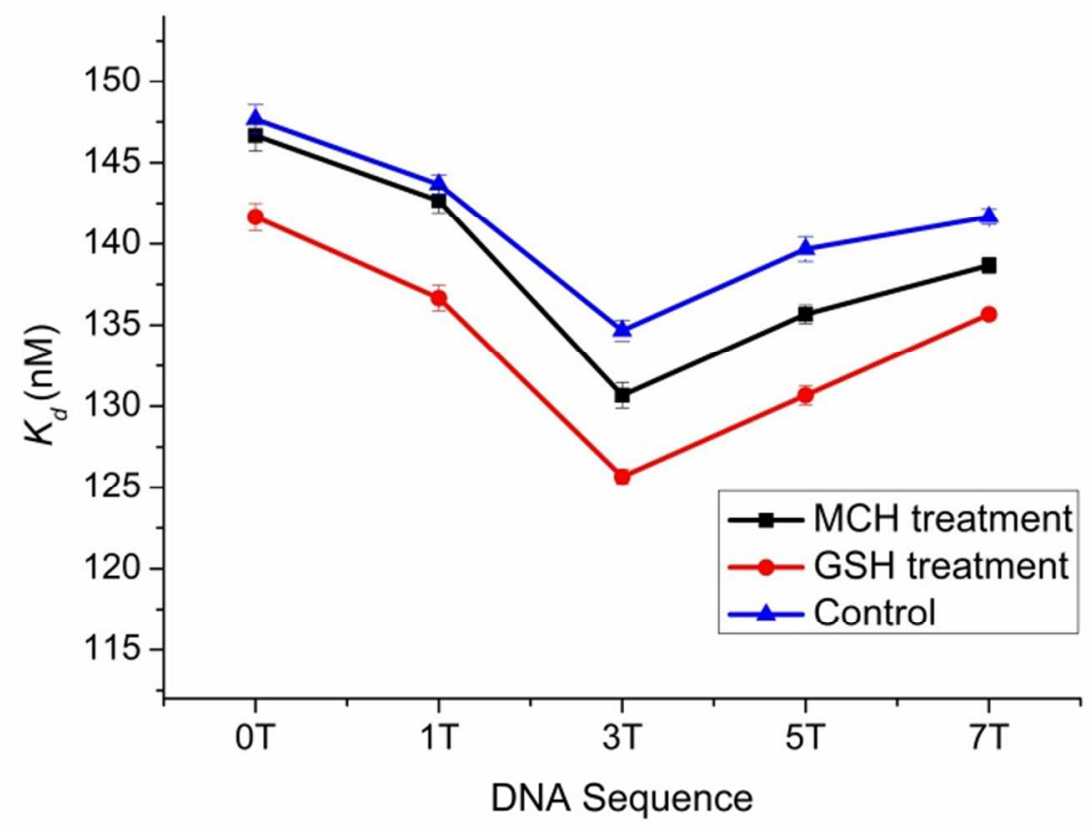

Figure S7. Comparison of SNAs with and without the treatment by GSH or MCH regarding the binding constants of SH-DNAs with various spacer sequences towards their complementary DNAs on each AuNP.
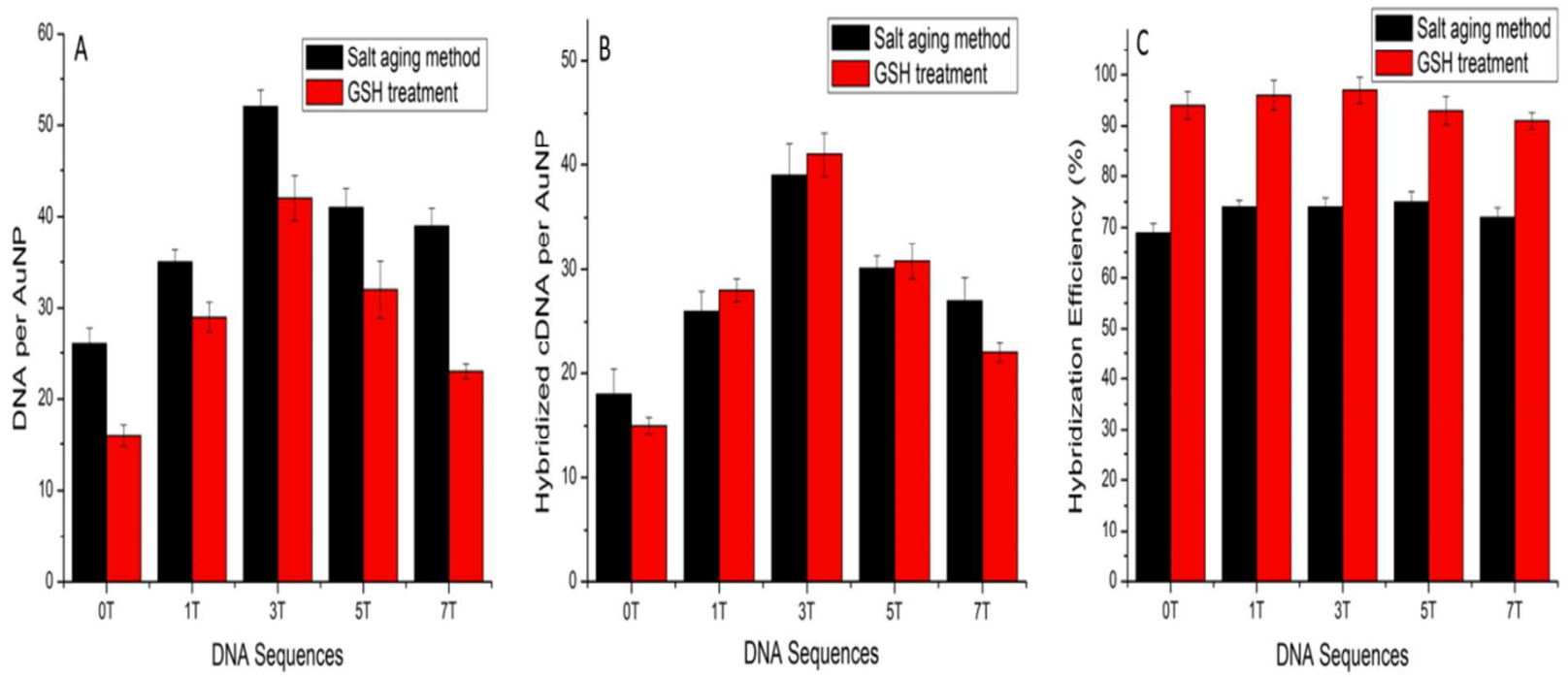

Figure S8. (A) The DNA loading capacity, (B) the number of hybridized cDNA (DNA16), and (C) the hybridization efficiency of the SNAs (AuNPs functionalized with DNA 1-5, respectively) prepared using the salt-aging method with and without GSH treatment. 


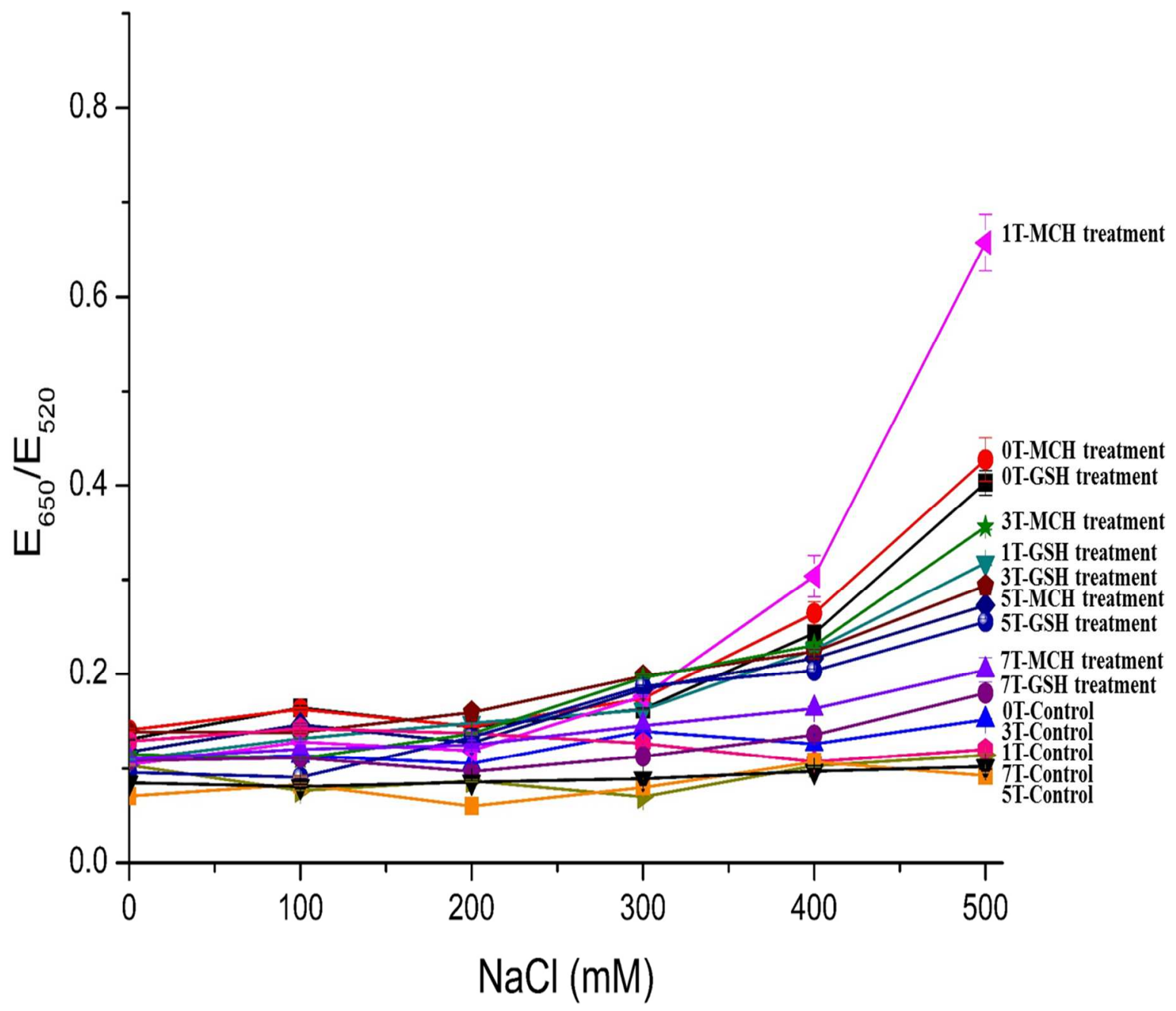

Figure S9. Extinction ratio $\mathrm{E}_{650} / \mathrm{E}_{520}$ as a function of $\mathrm{NaCl}$ concentration for AuNP-templated SNAs synthesized from DNAs 1-5. 
Table S1. Comparison of the hybridization efficiency $(h \%)$ of the two SNAs (Salt-agingSNAs $V s$ pH3-SNAs) towards the complementary sequence (DNA 16 in Table 1) under different $\mathrm{NaCl}$ concentrations (150 mM Vs $400 \mathrm{mM})$.

\section{A. pH3-SNAs}

\begin{tabular}{|c|c|c|c|c|c|}
\hline$[\mathrm{NaCl}]$ & $0 \mathrm{~T}$ & $1 \mathrm{~T}$ & $3 \mathrm{~T}$ & $5 \mathrm{~T}$ & $7 \mathrm{~T}$ \\
\hline$h \%$ with $150 \mathrm{mM}$ & $45 \% \pm 2.6 \%$ & $46 \% \pm 3.0 \%$ & $48 \% \pm 2.9 \%$ & $50 \% \pm 1.6 \%$ & $51 \% \pm 2.3 \%$ \\
\hline$h \%$ with $400 \mathrm{mM}$ & $61 \% \pm 2.8 \%$ & $64 \% \pm 2.2 \%$ & $67 \% \pm 3.1 \%$ & $67 \% \pm 1.3 \%$ & $69 \% \pm 1.9 \%$ \\
\hline$\Delta h \%$ & $16 \% \pm 1.3 \%$ & $18 \% \pm 1.5 \%$ & $19 \% \pm 1.6 \%$ & $17 \% \pm 0.5 \%$ & $18 \% \pm 0.9 \%$ \\
\hline Significant difference & Yes, $<0.05$ & Yes, $<0.05$ & Yes, $<0.05$ & Yes, $<0.05$ & Yes, $<0.05$ \\
\hline
\end{tabular}

B. Salt-aging-SNAs

\begin{tabular}{|c|c|c|c|c|c|}
\hline$[\mathrm{NaCl}]$ & $0 \mathrm{~T}$ & $1 \mathrm{~T}$ & $3 \mathrm{~T}$ & $5 \mathrm{~T}$ & $7 \mathrm{~T}$ \\
\hline$h \%$ with $150 \mathrm{mM}$ & $76 \% \pm 1.9 \%$ & $78 \% \pm 2.1 \%$ & $79 \% \pm 2.7 \%$ & $80 \% \pm 1.9 \%$ & $80 \% \pm 1.4 \%$ \\
\hline$h \%$ with $400 \mathrm{mM}$ & $79 \% \pm 2.4 \%$ & $81 \% \pm 1.5 \%$ & $81 \% \pm 2.3 \%$ & $82 \% \pm 1.6 \%$ & $84 \% \pm 0.9 \%$ \\
\hline$\Delta h \%$ & $3 \% \pm 0.8 \%$ & $3 \% \pm 1.1 \%$ & $2 \% \pm 0.9 \%$ & $2 \% \pm 0.7 \%$ & $4 \% \pm 0.5 \%$ \\
\hline Significant difference & No & No & No & No & No \\
\hline
\end{tabular}

